Pacific

Journal of

Mathematics

\title{
AN ABSOLUTE ESTIMATE OF THE HOMOGENEOUS EXPANSIONS OF HOLOMORPHIC MAPPINGS
}

TAISHUN LIU AND JIANFEI WANG 


\title{
AN ABSOLUTE ESTIMATE OF THE HOMOGENEOUS EXPANSIONS OF HOLOMORPHIC MAPPINGS
}

\author{
TAISHUN LIU AND JIANFEI WANG
}

Let $f: \Omega \rightarrow \Omega$ be a holomorphic mapping, where $\Omega$ is one of the four classical domains in $\mathbb{C}^{m \times n}$. We show that, if $P=f(0)$, we have

$$
\sum_{k=0}^{\infty} \frac{\left\|D \varphi_{P}(P)\left[D^{k} f(0)\left(Z^{k}\right)\right]\right\|_{\Omega}}{k !\left\|D \varphi_{P}(P)\right\|}<1
$$

for $\|Z\|_{\Omega}<\frac{1}{3}$ and $\varphi_{P} \in$ Aut $\Omega$ such that $\varphi_{P}(P)=0$. This generalizes to higher dimensions a classical result of Bohr, which corresponds to the case $\Omega=\{z:|z|<1\} \subset \mathbb{C}$. The constant $\frac{1}{3}$ is the best possible.

Let $f$ be a holomorphic function from the unit disc $D \subset \mathbb{C}$ to itself, with Taylor expansion

$$
f(z)=\sum_{k=0}^{\infty} a_{k} z^{k}
$$

Then

$$
\sum_{k=0}^{\infty}\left|a_{k} z^{k}\right|<1 \quad \text { for }|z|<\frac{1}{3} .
$$

This result, known as Bohr's theorem, was originally obtained in [Bohr 1914] for $|z|<\frac{1}{6}$. That $\frac{1}{6}$ can be improved to $\frac{1}{3}$ and that this is the best possible constant was quickly realized independently by M. Riesz, I. Schur, and N. Wiener. New proofs were given in [Sidon 1927; Tomić 1962]. More recently, attention has been paid to multidimensional generalizations of Bohr's theorem [Boas and Khavinson 1997; Boas 2000; Defant et al. 2003; Dineen and Timoney 1989; 1991]. Such generalizations were obtained by studying the power series of a holomorphic function defined in

$$
B_{\ell_{p}^{n}}:=\left\{z \in \mathbb{C}^{n}:\|z\|_{p}=\left(\sum_{k=1}^{n}\left|z_{k}\right|^{p}\right)^{1 / p}<1\right\}
$$

MSC2000: primary 30H05; secondary 32A05.

Keywords: holomorphic mapping, homogeneous expansion, classical domains, Bohr's theorem. This work was supported by the National Natural Science Foundation of China Grant No. 10571164 and Specialized Research Fund for the Doctoral Program of Higher Education (SRFDP) Grant No. 20050358052. 
with modulus less than 1 . They can be summarized as follows:

$$
\begin{aligned}
\frac{1}{3 \sqrt[3]{e}} \frac{1}{n^{1-1 / p}} & \leq K<3\left(\frac{\log n}{n}\right)^{1-1 / p} & & \text { if } 1 \leq p \leq 2, \\
\frac{1}{3} \frac{1}{\sqrt{n}} & \leq K<2 \sqrt{\frac{\log n}{n}} & & \text { if } 2 \leq p \leq \infty,
\end{aligned}
$$

where $K$ is the supremum of $r \in[0,1]$ such that $\sum_{\alpha \geq 0}\left|c_{\alpha} z^{\alpha}\right|<1$ for $z \in r B_{\ell_{p}^{n}}$ whenever $\left|\sum_{\alpha \geq 0} c_{\alpha} z^{\alpha}\right|<1$ for $z \in B_{\ell_{p}^{n}}$. Here the sum is taken over multi-indices $\alpha=\left(\alpha_{1}, \alpha_{2}, \ldots, \alpha_{n}\right)$, where the $\alpha_{j}$ are nonnegative integers. Aizenberg [2000, Theorem 9] established these inequalities for $p=1$. Dineen and Timoney [1989] investigated the case $p=\infty$ and their result was clarified in [Boas and Khavinson 1997]. Boas [2000, Theorem 3] then generalized to $1<p<\infty$.

The result of Aizenberg and Boas does not, strictly speaking, reduce to Bohr's classical theorem, as consideration of the case $n=1$ shows. In this article, we give a new generalization of Bohr's theorem to higher dimensions. We investigate holomorphic mappings from $\Omega$ to $\Omega$, where $\Omega$ is one of the four classical domains in $\mathbb{C}^{n}$ (see below), and demonstrate a result analogous to Bohr's, which reduces to it when $n=1$. We also prove that the constant $\frac{1}{3}$ is best possible in higher dimensions. In the proof we use homogeneous expansions of holomorphic mappings, which replace multiple power series. The Minkowski norm in each of the four classical domains replaces the Euclidean norm, and certain properties of the automorphisms of these domains play an important role.

We first recall the definition of the four classical domains in the sense of Hua [1963]. Let $\mathbb{C}^{m \times n}$ denote the set of $m \times n$ matrices $Z=\left(z_{i j}\right)_{1 \leq i \leq m, 1 \leq j \leq n}$, with $z_{i j} \in \mathbb{C}$ and $1 \leq m \leq n$; denote by $Z^{\prime}$ and $\bar{Z}$, respectively, the transpose and the complex conjugate of $Z$.

The first classical domain, $\mathscr{R}_{I}(m, n) \subset \mathbb{C}^{m \times n}$, consists of matrices $Z$ such that $I_{m}-Z \bar{Z}^{\prime}>0$, where $I_{m}$ is the identity matrix of rank $m$ and the inequality sign means that the left-hand side is positive definite.

The second classical domain, $\mathscr{R}_{I I}(n) \subset \mathbb{C}^{n \times n}$, consists of $Z$ such that $Z=Z^{\prime}$ and $I_{n}-Z \bar{Z}^{\prime}>0$.

The third classical domain, $\mathscr{R}_{I I I}(n) \subset \mathbb{C}^{n \times n}$, consists of $Z$ such that $Z=-Z^{\prime}$ and $I_{n}-Z \bar{Z}^{\prime}>0$.

The fourth classical domain, $\mathscr{R}_{I V}(n) \subset \mathbb{C}^{n}$, is the set of $Z=\left(z_{1}, z_{2}, \ldots, z_{n}\right)$ satisfying

$$
\left|Z Z^{\prime}\right|^{2}+1-2|Z|^{2}>0, \quad\left|Z Z^{\prime}\right|<1 .
$$

Let $\Omega$ denote one of the four classical domains or the unit polydisc $D^{n} \subset \mathbb{C}^{n}$. The span of $\Omega$ in the ambient space $\left(\mathbb{C}^{m \times n}, \mathbb{C}^{n \times n}\right.$ or $\mathbb{C}^{n}$, as the case may be) is provided with a Minkowski functional $\|\cdot\|_{\Omega}$ arising from $\Omega$ [Liu and Ren 1998]. 
By results in [Liu 1989] and [Gong 1998], we know that

$$
\|Z\|_{\Omega}=\sup \left\{\left|\alpha Z \beta^{\prime}\right|: \alpha \in \partial B^{m}, \beta \in \partial B^{n}\right\}
$$

if $\Omega=\mathscr{R}_{I}(m, n), \mathscr{R}_{I I}(n)$, or $\mathscr{R}_{I I I}(n)$ (with $m=n$ in the latter two cases), and this supremum equals the square root of the largest characteristic root of $Z \bar{Z}^{\prime}$; if $\Omega=D^{n}$, then $\|Z\|_{\Omega}=\max \left\{\left|z_{k}\right|: 1 \leq k \leq n\right\}$; and if $\Omega=\mathscr{R}_{I V}(n)$, then

$$
\|Z\|_{\Omega}=\sqrt{|Z|^{2}+\sqrt{|Z|^{4}-\left|Z Z^{\prime}\right|^{2}}},
$$

where $|Z|$ is the Euclidean norm in $\mathbb{C}^{n}$. Hence $\Omega=\mathscr{R}_{I}(m, n)$ is the unit ball of the complex Banach space $\mathbb{C}^{m \times n}$ with respect to the norm $\|\cdot\|_{\Omega}$. The subspaces $\left\{Z \in \mathbb{C}^{n \times n}: Z=Z^{\prime}\right\}$ and $\left\{Z \in \mathbb{C}^{n \times n}: Z=-Z^{\prime}\right\}$ are complex Banach spaces with respect to the norm $\|\cdot\|_{\Omega}$, for $\Omega=\mathscr{R}_{I I}(n)$ and $\mathscr{R}_{I I I}(n)$ respectively, and $\Omega$ is the unit ball for that norm. $\mathbb{C}^{n}$ is a complex Banach space whose unit ball is $\Omega=\mathscr{R}_{I V}(n)$ for the norm $\|\cdot\|_{\Omega}$.

Let $\partial \Omega$ and $\partial_{0} \Omega$ denote the topological boundary and distinguished boundary of $\Omega$. Denote by $H(\Omega, \Omega)$ the space of holomorphic mappings from $\Omega$ to $\Omega$, and by Aut $\Omega$ the group of holomorphic automorphisms of $\Omega$. Let $\bar{\Omega}$ denote the closure of $\Omega$. If $T$ is a linear operator between normed linear spaces, we denote by $\|T\|$ its norm. Finally, $D^{k} f(Z)$ will mean the $k$-th Fréchet derivative of $f$ at $Z$, where $f \in H(\Omega, \Omega)$ and $k$ is a nonnegative integer.

Theorem. Let $f: \Omega \rightarrow \Omega$ be holomorphic, where $\Omega$ is one of the classical domains, and set $P=f(0)$. Then

$$
\sum_{k=0}^{\infty} \frac{\left\|D \varphi_{P}(P)\left[D^{k} f(0)\left(Z^{k}\right)\right]\right\|_{\Omega}}{k !\left\|D \varphi_{P}(P)\right\|}<1
$$

for $\|Z\|_{\Omega}<\frac{1}{3}$ and $\varphi_{P} \in$ Aut $\Omega$ such that $\varphi_{P}(P)=0$.

If $\|Z\|_{\Omega}>\frac{1}{3}$, there exists a holomorphic map $f: \Omega \rightarrow \Omega$ such that (1) is not valid.

As already mentioned, if $\Omega=D \subset \mathbb{C}$, inequality (1) reduces to the relation (0) of page 155, recovering Bohr's classical theorem in one complex variable.

The proof of the theorem requires some lemmas, the first two of which are well known.

Lemma 1 [Liu 1989]. Let $P \in \mathscr{R}_{I}(m, n)$. There is an $m \times m$ unitary matrix $U$ and an $n \times n$ unitary matrix $V$ for which $P$ has the polar decomposition

$$
P=U\left(\begin{array}{cccccc}
\lambda_{1} & \cdots & 0 & 0 & \cdots & 0 \\
\vdots & \ddots & \vdots & \vdots & \ddots & \vdots \\
0 & \cdots & \lambda_{m} & 0 & \cdots & 0
\end{array}\right) V
$$


where $1>\lambda_{1} \geq \lambda_{2} \geq \cdots \geq \lambda_{m} \geq 0$ and $\|P\|_{\Omega}=\lambda_{1}$. Set

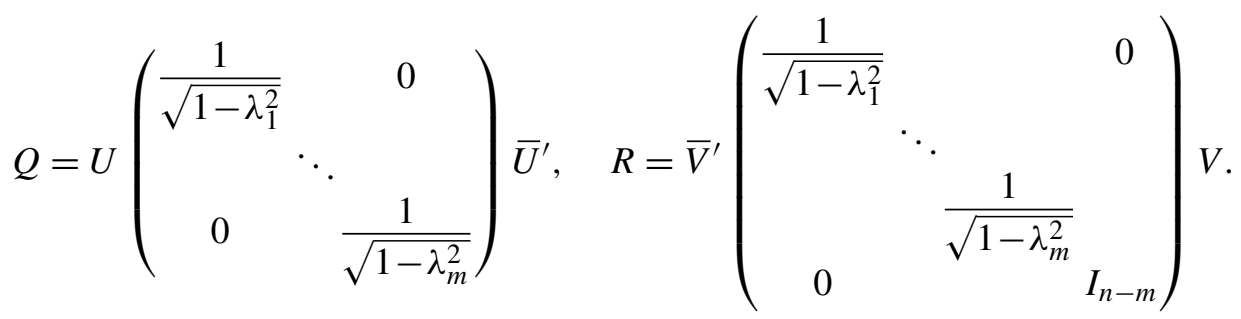

Then

$$
\varphi_{P}(Z)=Q^{-1}\left(I_{m}-Z \bar{P}^{\prime}\right)^{-1}(P-Z) R \in \operatorname{Aut} \mathscr{R}_{I}(m, n)
$$

for $Z \in \overline{\mathscr{R}_{I}(m, n)}$, and hence

$$
D \varphi_{P}(P)(W)=-Q W R
$$

for $W \in \mathbb{C}^{m \times n}$.

Lemma 2 [Liu 1989]. Given any $A \in \mathscr{R}_{I V}(n)$, there exist a real orthogonal $n \times n$ matrix $T$ and $1>\lambda_{1} \geq \lambda_{2} \geq 0$ such that

$$
A=e^{i \theta}\left(\frac{\lambda_{1}+\lambda_{2}}{2}, i \frac{\lambda_{1}-\lambda_{2}}{2}, 0, \ldots, 0\right) T \in \mathscr{R}_{I V}(n)
$$

and $\|A\|_{\Omega}=\lambda_{1}$, where $i=\sqrt{-1}$ and $\theta \in \mathbb{R}$. Let

$$
Q=T^{\prime}\left(\begin{array}{ccc}
1+\lambda_{1} \lambda_{2} & 0 & 0 \\
0 & 1-\lambda_{1} \lambda_{2} & 0 \\
0 & 0 & \sqrt{\left(1-\lambda_{1}^{2}\right)\left(1-\lambda_{2}^{2}\right)} I_{n-2}
\end{array}\right) T .
$$

Then

$$
\varphi_{A}(Z)=\frac{A+Z Z^{\prime} \bar{A}-Z Q}{1-2 Z \bar{A}^{\prime}+Z Z^{\prime} \bar{A} \bar{A}^{\prime}} \in \operatorname{Aut} \mathscr{R}_{I V}(n)
$$

for any $Z \in \overline{\mathscr{R}_{I V}(n)}$, and hence

$$
D \varphi_{A}(A)(X)=X \frac{2 A^{\prime} \bar{A}-Q}{1-2|A|^{2}+\left|A A^{\prime}\right|^{2}}
$$

for $X \in \mathbb{C}^{n}$.

Lemma 3. Let $\Omega$ be one of the four classical domains. Then

$$
\left\|D \varphi_{P}(P)\right\|=\frac{1}{1-\|P\|_{\Omega}^{2}}
$$

for any $P \in \Omega$. 
Proof. Case 1: $\Omega$ is one of $\mathscr{R}_{I}(m, n), \mathscr{R}_{I I}(n), \mathscr{R}_{I I I}(n)$. We assume without loss of generality that $\Omega=\mathscr{R}_{I}(m, n)$. From Lemma 1 and the definition of $\|\cdot\|_{\Omega}$, we get

$$
\begin{aligned}
\left\|D \varphi_{P}(P)(W)\right\|_{\Omega} & =\sup \left\{\left|\alpha Q W R \beta^{\prime}\right|: \alpha \in \partial B^{m}, \beta \in \partial B^{n}\right\} \\
& \leq \sup \left\{\frac{\left|\alpha W \beta^{\prime}\right|}{1-\lambda_{1}^{2}}: \alpha \in \partial B^{m}, \beta \in \partial B^{n}\right\}=\frac{\|W\|_{\Omega}}{1-\lambda_{1}^{2}}
\end{aligned}
$$

for $W \in \mathbb{C}^{m \times n}$. This implies that

$$
\left\|D \varphi_{P}(P)\right\|_{\Omega} \leq \frac{1}{1-\lambda_{1}^{2}}=\frac{1}{1-\|P\|_{\Omega}^{2}} .
$$

If we take $Z_{0} \in \overline{\mathscr{R}_{I}(m, n)}$ with $\left\|Z_{0}\right\|_{\Omega}=1$ such that

$$
\bar{U}^{\prime} Z_{0} \bar{V}^{\prime}=\left(\begin{array}{cccc}
1 & 0 & & 0 \\
0 & 0 & & 0 \\
& & \ddots & \\
0 & 0 & & 0
\end{array}\right)
$$

we obtain

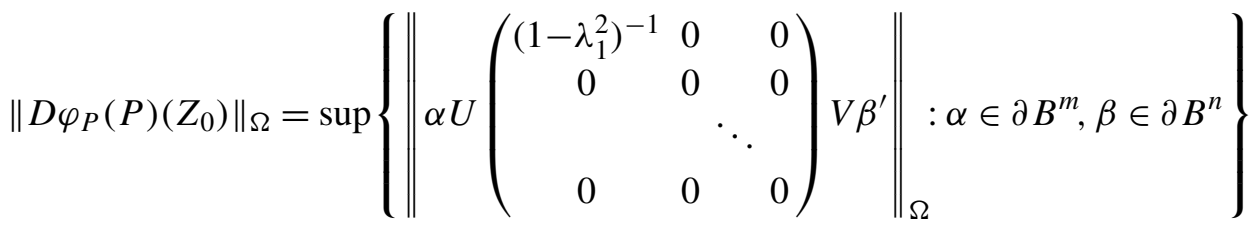

$$
\begin{aligned}
& =\frac{1}{1-\lambda_{1}^{2}} \text {. }
\end{aligned}
$$

This shows that $\left\|D \varphi_{P}(P)\right\|_{\Omega} \geq \frac{1}{1-\lambda_{1}^{2}}=\frac{1}{1-\|P\|_{\Omega}^{2}}$, which leads to the desired
conclusion.

Case 2: $\Omega=\mathscr{R}_{I V}(n)$. Taking $A=P \in \Omega$ in Lemma 2 and expressing it as in (2), we see from the lemma that

$$
D \varphi_{A}(A)(Z)=\frac{W}{1-2|A|^{2}+\left|A A^{\prime}\right|^{2}},
$$

where $W=Z\left(2 A^{\prime} \bar{A}-Q\right)$, with $Q$ as in (3). It is clear that

$$
1-2|A|^{2}+\left|A A^{\prime}\right|=\left(1-\lambda_{1}^{2}\right)\left(1-\lambda_{2}^{2}\right) \text {, }
$$

where $1>\lambda_{1} \geq \lambda_{2} \geq 0$, and that

$$
W=Z T^{\prime}\left(\begin{array}{ccc}
\frac{1}{2}\left(\lambda_{1}^{2}+\lambda_{2}^{2}-2\right) & -\frac{i}{2}\left(\lambda_{1}^{2}-\lambda_{2}^{2}\right) & 0 \\
\frac{i}{2}\left(\lambda_{1}^{2}-\lambda_{2}^{2}\right) & \frac{1}{2}\left(\lambda_{1}^{2}+\lambda_{2}^{2}-2\right) & 0 \\
0 & 0 & -\sqrt{\left(1-\lambda_{1}^{2}\right)\left(1-\lambda_{2}^{2}\right)} I_{n-2}
\end{array}\right) T .
$$


If $Z \in \partial_{0} \Omega$, that is, $Z=e^{i \theta}\left(x_{1}, x_{2}, \ldots, x_{n}\right)$ with $x_{k} \in \mathbb{R}$ for $k=1,2, \ldots, n$, it is clear that

$$
|Z|^{2}=\left|Z Z^{\prime}\right|=x_{1}^{2}+\cdots+x_{n}^{2}=1 .
$$

A simple computation then shows that

$$
\begin{aligned}
& W \bar{W}^{\prime}= \\
& Z T^{\prime}\left(\begin{array}{ccc}
\frac{1}{2}\left(\left(1-\lambda_{1}^{2}\right)^{2}+\left(1-\lambda_{2}^{2}\right)^{2}\right) & 0 & 0 \\
0 & \frac{1}{2}\left(\left(1-\lambda_{1}^{2}\right)^{2}+\left(1-\lambda_{2}^{2}\right)^{2}\right) & 0 \\
0 & 0 & \left(1-\lambda_{1}^{2}\right)\left(1-\lambda_{2}^{2}\right) I_{n-2}
\end{array}\right) T \bar{Z}^{\prime} .
\end{aligned}
$$

Since $T$ is a real orthogonal matrix we obtain

$$
|W|^{2}=W \bar{W}^{\prime} \leq \frac{\left(1-\lambda_{1}^{2}\right)^{2}+\left(1-\lambda_{2}^{2}\right)^{2}}{2}\left|Z \bar{Z}^{\prime}\right|=\frac{\left(1-\lambda_{1}^{2}\right)^{2}+\left(1-\lambda_{2}^{2}\right)^{2}}{2},
$$

where we have used the Schwarz inequality on the coefficient of $I_{n-2}$. Clearly,

$$
W W^{\prime}=\left(1-\lambda_{1}^{2}\right)\left(1-\lambda_{2}^{2}\right) Z Z^{\prime} .
$$

Hence

$$
\|W\|_{\Omega}=\sqrt{|W|^{2}+\sqrt{|W|^{4}-\left|W W^{\prime}\right|^{2}}} \leq 1-\lambda_{2}^{2},
$$

which together with (4) and (5) yields

$$
\left\|D \varphi_{A}(A)(Z)\right\|_{\Omega} \leq \frac{1}{1-\lambda_{1}^{2}} \quad \text { for } Z \in \partial_{0} \Omega .
$$

If $Z \in \partial \Omega$, there exists a linear functional $f$ satisfying

$$
f(Z)=\left\|D \varphi_{A}(A)(Z)\right\|_{\Omega}, \quad\|f\|=1 .
$$

The function $g$ defined by $g(\xi)=f\left(D \varphi_{A}(A)(\xi)\right)$ is holomorphic on $\bar{\Omega}$, so we obtain from the preceding inequality

$$
|g(\xi)| \leq\left\|D \varphi_{A}(A)(\xi)\right\|_{\Omega} \leq \frac{1}{1-\lambda_{1}^{2}} \quad \text { for } \xi \in \partial_{0} \Omega .
$$

On the other hand, the maximum principle gives

$$
|g(Z)|=\left\|D \varphi_{A}(A)(Z)\right\|_{\Omega} \leq\left\|D \varphi_{A}(A)(\xi)\right\|_{\Omega} \leq \frac{1}{1-\lambda_{1}^{2}} \quad \text { for } \xi \in \partial_{0} \Omega .
$$

Therefore

$$
\left\|D \varphi_{A}(A)\right\| \leq \frac{1}{1-\lambda_{1}^{2}}=\frac{1}{1-\|A\|_{\Omega}^{2}}
$$


There remains to show the reverse inequality,

$$
\left\|D \varphi_{A}(A)\right\| \geq \frac{1}{1-\|A\|_{\Omega}^{2}} .
$$

Take $Z_{0}=(1,0,0, \ldots, 0) \in \partial_{0} \Omega$. Then $\left\|Z_{0}\right\|_{\Omega}=1$, and

$$
\left\|D \varphi_{A}(A)\left(Z_{0}\right)\right\|_{\Omega}=\frac{1}{1-\lambda_{1}^{2}}=\frac{1}{1-\|A\|_{\Omega}^{2}} .
$$

But this immediately implies (7), completing the proof.

Proof of the Theorem. Case 1: $\Omega=D^{n}$. For $\|Z\|_{\Omega}<\frac{1}{3}$ it is easy to show that

$$
\sum_{k=0}^{\infty} \frac{\left\|D \varphi_{P}(P)\left[D^{k} f(0)\left(Z^{k}\right)\right]\right\|_{\Omega}}{k !\left\|D \varphi_{P}(P)\right\|}<1 .
$$

On the other hand, when $\|Z\|_{\Omega}>\frac{1}{3}$, we let $\left\|Z_{0}\right\|_{\Omega}=\left|z_{j}^{0}\right|=\max _{k}\left\{\left|z_{k}^{0}\right|\right\}>\frac{1}{3}$ and define

$$
f(Z)=\frac{p_{j}-z_{j}}{1-p_{j} z_{j}}
$$

where $1>p_{j}>\frac{1}{2}\left(\left|z_{j}^{0}\right|^{-1}-1\right)$. Then

$$
\sum_{k=0}^{\infty} \frac{\left\|D \varphi_{P}(P)\left[D^{k} f(0)\left(Z_{0}^{k}\right)\right]\right\|_{\Omega}}{k !\left\|D \varphi_{P}(P)\right\|}>1 .
$$

Case 2: $\Omega$ is one of $\mathscr{R}_{I}(m, n), \mathscr{R}_{I I}(n), \mathscr{R}_{I I I}(n)$. We assume without loss of generality that $\Omega=\mathscr{R}_{I}(m, n)$. Take $P=f(0) \in \Omega$ and express it as in Lemma 1, defining $Q$ and $R$ accordingly. The lemma then says that

$$
\varphi_{P}(Z)=Q^{-1}\left(I_{m}-Z \bar{P}^{\prime}\right)^{-1}(P-Z) R
$$

for any $Z \in \mathscr{R}_{I}(m, n)$. From Lemma 3, we get

$$
\left\|D \varphi_{P}(P)\right\|_{\Omega}=\frac{1}{1-\|P\|_{\Omega}^{2}} .
$$

Since $\Omega$ is a convex domain, for a fixed $k$ we can define

$$
f_{k}(Z)=\sum_{j=1}^{k} \frac{f\left(e^{i 2 \pi j / k} Z\right)}{k} .
$$

Then $f_{k} \in H(\Omega, \Omega)$. It is clear that

$$
\frac{1}{k} \sum_{j=1}^{k} e^{i 2 \pi j l / k}= \begin{cases}1 & \text { if } l \equiv 0 \quad(\bmod k) \\ 0 & \text { otherwise }\end{cases}
$$


From the homogeneous expansion of the holomorphic mapping $f$, we get

$$
f_{k}(Z)=\frac{1}{k}\left(\sum_{j=1}^{k}\left(f(0)+\sum_{l=1}^{\infty} e^{i 2 \pi j l / k} \frac{D^{l} f(0)\left(Z^{l}\right)}{l !}\right)\right) .
$$

This implies that

$$
\begin{aligned}
\varphi_{P} \circ f_{k}(Z) & =\varphi_{P}\left(P+\sum_{l=1}^{\infty} \frac{D^{l k} f(0)\left(Z^{l k}\right)}{(l k) !}\right) \\
& =\varphi_{P}(P)+D \varphi_{P}(P)\left(\sum_{l=1}^{\infty} \frac{D^{l k} f(0)\left(Z^{l k}\right)}{(l k) !}\right)+\cdots \\
& =\frac{D \varphi_{P}(P)\left[D^{k} f(0)\left(Z^{k}\right)\right]}{k !}+\frac{D \varphi_{P}(P)\left[D^{2 k} f(0)\left(Z^{2 k}\right)\right]}{(2 k) !}+\cdots,
\end{aligned}
$$

and hence

$$
\frac{D \varphi_{P}(P)\left[D^{k} f(0)\left(Z^{k}\right)\right]}{k !}=\frac{1}{2 \pi} \int_{0}^{2 \pi} \varphi_{P} \circ f_{k}\left(Z e^{i \theta}\right) e^{-i k \theta} d \theta
$$

since $\varphi_{P} \circ f_{k}$ is holomorphic and maps 0 to 0 . Again because $\varphi_{P} \circ f_{k} \in H(\Omega, \Omega)$, we have

$$
\frac{\left\|D \varphi_{P}(P)\left[D^{k} f(0)\left(Z^{k}\right)\right]\right\|_{\Omega}}{k !}<1
$$

for any $Z \in \Omega$. Thus

$$
\frac{\left\|D \varphi_{P}(P)\left[D^{k} f(0)\left(Z^{k}\right)\right]\right\|_{\Omega}}{k !} \leq 1
$$

for any $Z \in \bar{\Omega}$. This shows that

$$
\frac{\left\|D \varphi_{P}(P)\left[D^{k} f(0)\left(Z^{k}\right)\right]\right\|_{\Omega}}{k !}=\|Z\|_{\Omega}^{k} \frac{\left\|D \varphi_{P}(P)\left[D^{k} f(0)\left(Z^{k} /\|Z\|^{k}\right)\right]\right\|_{\Omega}}{k !} \leq\|Z\|_{\Omega}^{k} .
$$

Using the equality $\left\|D \varphi_{P}(P)\right\|=\frac{1}{1-\|P\|_{\Omega}^{2}}$ from Lemma 3, we then get

$$
\begin{aligned}
\sum_{k=0}^{\infty} \frac{\left\|D \varphi_{P}(P)\left[D^{k} f(0)\left(Z^{k}\right)\right]\right\|_{\Omega}}{k !\left\|D \varphi_{P}(P)\right\|} & \leq\|P\|_{\Omega}+\left(1-\|P\|_{\Omega}^{2}\right) \sum_{k=1}^{\infty}\|Z\|_{\Omega}^{k} \\
& <\|P\|_{\Omega}+\left(1-\|P\|_{\Omega}^{2}\right) \sum_{k=1}^{\infty}\left(\frac{1}{3}\right)^{k} \\
& =\|P\|_{\Omega}+\frac{1-\|P\|_{\Omega}^{2}}{2}=1-\frac{\left(1-\|P\|_{\Omega}\right)^{2}}{2}<1
\end{aligned}
$$

for $\|Z\|_{\Omega}<\frac{1}{3}$. 
There remains to show that $\frac{1}{3}$ is the best possible constant. In fact, if $Z \in \Omega$ with $\|Z\|_{\Omega}>\frac{1}{3}$, we take

$$
Z_{0}=\left(\begin{array}{cccccc}
\mu_{1} & \cdots & 0 & 0 & \cdots & 0 \\
\vdots & \ddots & \vdots & \vdots & \ddots & \vdots \\
0 & \cdots & \mu_{m} & 0 & \cdots & 0
\end{array}\right) \in \mathscr{R}_{I}(m, n)
$$

where $1>\mu_{1} \geq \mu_{2} \geq \cdots \geq \mu_{m} \geq 0$ and $\left\|Z_{0}\right\|_{\Omega}=\mu_{1}>\frac{1}{3}$. Take $p_{11} \in \mathbb{R}$ such that

$$
\frac{1}{2}\left(\frac{1}{\mu_{1}}-1\right)<p_{11}<1 .
$$

If we define $f \in H(\Omega, \Omega)$ by

$$
f(Z)=\left(\begin{array}{cccc}
\frac{p_{11}-z_{11}}{1-p_{11} z_{11}} & 0 & & 0 \\
0 & 0 & & 0 \\
& & \ddots & \\
0 & 0 & & 0
\end{array}\right),
$$

we obtain successively

$$
\begin{aligned}
& P=f(0)=\left(\begin{array}{cccc}
p_{11} & 0 & & 0 \\
0 & 0 & & 0 \\
& & \ddots & \\
0 & 0 & & 0
\end{array}\right) \\
& Q=\left(\begin{array}{cc}
\left(1-p_{11}^{2}\right)^{-1 / 2} & 0 \\
0 & I_{m-1}
\end{array}\right), \quad R=\left(\begin{array}{cc}
\left(1-p_{11}^{2}\right)^{-1 / 2} & 0 \\
0 & I_{n-1}
\end{array}\right) \text {, } \\
& \left\|D \varphi_{P}(P) P\right\|_{\Omega}=\frac{p_{11}}{1-p_{11}^{2}}, \quad \frac{D^{k} f(0)\left(Z^{k}\right)}{k !}=\left(p_{11}^{2}-1\right) p_{11}^{k-1} z_{11}^{k}\left(\begin{array}{cccc}
1 & 0 & & 0 \\
0 & 0 & & 0 \\
& & \ddots & \\
0 & 0 & & 0
\end{array}\right)
\end{aligned}
$$

for $k \geq 1$. This implies that

$$
\frac{\left\|D \varphi_{P}(P)\left[D^{k} f(0)\left(Z_{0}^{k}\right)\right]\right\|_{\Omega}}{k !}=p_{11}^{k-1}\left|z_{11}\right|^{k}=p_{11}^{k-1} \mu_{1}^{k}
$$

when $k \geq 1$. In view of the definition of $Z_{0}$, we get

$$
\left\|D \varphi_{P}(P)\right\|=\frac{1}{1-p_{11}^{2}} .
$$


Therefore

$$
\begin{aligned}
\sum_{k=0}^{\infty} \frac{\left\|D \varphi_{P}(P)\left[D^{k} f(0)\left(Z_{0}^{k}\right)\right]\right\|_{\Omega}}{k !\left\|D \varphi_{P}(P)\right\|} & =p_{11}+\left(1-p_{11}^{2}\right) \sum_{k=1}^{\infty} p_{11}^{k-1} \mu_{1}^{k} \\
& =p_{11}+\left(1-p_{11}^{2}\right) \frac{\mu_{1}}{1-p_{11} \mu_{1}}
\end{aligned}
$$

Then we immediately get from (8) the desired inequality

$$
\sum_{k=0}^{\infty} \frac{\left\|D \varphi_{P}(P)\left[D^{k} f(0)\left(Z_{0}^{k}\right)\right]\right\|_{\Omega}}{k !\left\|D \varphi_{P}(P)\right\|}>1 .
$$

Case 3: $\Omega=\mathscr{R}_{I V}$. The proof of (1) for $\|Z\|_{\Omega}<\frac{1}{3}$ changes little from Case 2. To show that $\frac{1}{3}$ is best possible, let $Z_{0}=\left(\mu_{1}, 0, \ldots, 0\right) \in \mathscr{R}_{I V}(n)$, with $1>\mu_{1}>\frac{1}{3}$. We have $\left\|Z_{0}\right\|_{\Omega}=\mu_{1}$, by [Liu 1989]. Therefore

$$
Q=\left(\begin{array}{ccc}
1+\mu_{1}^{2} & 0 & 0 \\
0 & 1-\mu_{1}^{2} & 0 \\
0 & 0 & \left(1-\mu_{1}^{2}\right) I_{n-2}
\end{array}\right)
$$

Take $p_{11} \in \mathbb{R}$ with $\frac{1}{2}\left(\frac{1}{\mu_{1}}-1\right)<p_{11}<1$ and define

$$
f(Z)=\left(\frac{p_{11}-z_{1}}{1-p_{11} z_{1}}, 0, \ldots, 0\right) \in H(\Omega, \Omega) .
$$

Then $P=f(0)=\left(p_{11}, 0, \ldots, 0\right)$. From Lemma 2 we obtain

$$
\frac{\left\|D \varphi_{P}(P)\left[D^{k} f(0)\left(Z_{0}^{k}\right)\right]\right\|_{\Omega}}{k !}=p_{11}^{k-1}\left|\mu_{1}\right|^{k}
$$

when $k \geq 1$. Hence, as required,

$$
\begin{aligned}
\sum_{k=0}^{\infty} \frac{\left\|D \varphi_{P}(P)\left[D^{k} f(0)\left(Z_{0}^{k}\right)\right]\right\|_{\Omega}}{k !\left\|D \varphi_{P}(P)\right\|} & =p_{11}+\left(1-p_{11}^{2}\right) \sum_{k=1}^{\infty} p_{11}^{k-1} \mu_{1}^{k} \\
& =p_{11}+\left(1-p_{11}^{2}\right) \frac{\mu_{1}}{1-p_{11} \mu_{1}}>1
\end{aligned}
$$

From the proof of the theorem, we have obtained in addition:

Corollary. Let $P \in \Omega$ be given, where $\Omega$ is one of the four classical domains, and define

$$
\gamma_{1}=\frac{1}{2+\|P\|_{\Omega}}, \quad \gamma_{2}=\frac{1}{1+2\|P\|_{\Omega}} .
$$


If $f: \Omega \rightarrow \Omega$ is a holomorphic mapping taking 0 to $P$, the inequality

$$
\sum_{k=0}^{\infty} \frac{\left\|D \varphi_{P(P)}\left[D^{k} f(0)\left(Z^{k}\right)\right]\right\|_{\Omega}}{k !\left\|D \varphi_{P}(P)\right\|}<1
$$

holds for all $Z$ such that $\|Z\|_{\Omega}<\gamma_{1}$. If $\|Z\|_{\Omega}>\gamma_{2}$, there exists $f \in H(\Omega, \Omega)$ with $f(0)=P$ such that the inequality fails.

This leads naturally to the following problem:

Question. What is the best constant $\gamma_{P}$, depending on $\|P\|_{\Omega}$, such that

$$
\sum_{k=0}^{\infty} \frac{\left\|D \varphi_{P}(P)\left[D^{k} f(0)\left(Z^{k}\right)\right]\right\|_{\Omega}}{k !\left\|D \varphi_{P}(P)\right\|}<1
$$

whenever $\|Z\|_{\Omega}<\gamma_{P}$ ? According to the Corollary, $\gamma_{P} \in\left[\frac{1}{2+\|P\|_{\Omega}}, \frac{1}{1+2\|P\|_{\Omega}}\right]$.

\section{References}

[Aizenberg 2000] L. Aizenberg, "Multidimensional analogues of Bohr's theorem on power series", Proc. Amer. Math. Soc. 128:4 (2000), 1147-1155. MR 2000i:32001 Zbl 0948.32001

[Boas 2000] H. P. Boas, "Majorant series", J. Korean Math. Soc. 37:2 (2000), 321-337. Several complex variables (Seoul, 1998). MR 2001j:32001 Zbl 0965.32001

[Boas and Khavinson 1997] H. P. Boas and D. Khavinson, "Bohr's power series theorem in several variables", Proc. Amer. Math. Soc. 125:10 (1997), 2975-2979. MR 98i:32002 Zbl 0888.32001

[Bohr 1914] H. Bohr, “A theorem concerning power series", Proc. London. Math. Soc. (2) 13 (1914), 1-5. JFM 44.0289.01

[Defant et al. 2003] A. Defant, D. García, and M. Maestre, "Bohr's power series theorem and local Banach space theory", J. Reine Angew. Math. 557 (2003), 173-197. MR 2004d:46012 Zbl 1031. 46014

[Dineen and Timoney 1989] S. Dineen and R. M. Timoney, "Absolute bases, tensor products and a theorem of Bohr”, Studia Math. 94:3 (1989), 227-234. MR 91e:46006 Zbl 0713.46005

[Dineen and Timoney 1991] S. Dineen and R. M. Timoney, "On a problem of H. Bohr", Bull. Soc. Roy. Sci. Liège 60:6 (1991), 401-404. MR 93e:46050 Zbl 0795.46034

[Gong 1998] S. Gong, Convex and starlike mappings in several complex variables, Mathematics and its Applications 435, Kluwer, Dordrecht, 1998. MR 2000c:32054 Zbl 0926.32007

[Hua 1963] L. K. Hua, Harmonic analysis of functions of several complex variables in the classical domains, Translations of Mathematical Monographs 6, Amer. Math. Soc., Providence, RI, 1963. MR 30 \#2162 Zbl 0507.32025

[Liu 1989] T. S. Liu, The growth theorems and covering theorems for biholomorphic mappings on classical domains, Dissertation, University of Science and Technology of China, Hefei, 1989. In Chinese.

[Liu and Ren 1998] T. Liu and G. Ren, "Decomposition theorem of normalized biholomorphic convex mappings", J. Reine Angew. Math. 496 (1998), 1-13. MR 99e:32034 Zbl 0894.32009

[Sidon 1927] S. Sidon, "Über einen Satz von Herrn Bohr”, Math. Z. 26:1 (1927), 731-732. MR 1544888 JFM 53.0281 .04 
[Tomić 1962] M. Tomić, "Sur un théorème de H. Bohr", Math. Scand. 11 (1962), 103-106. MR 31 \#316 Zbl 0109.30202

Received December 23, 2005. Revised September 25, 2006.

TAISHUN LIU

Department of MATHEMATiCs

HuZHou TEACHERs College

HUZHOU, ZHEJIANG, 313000

CHINA

lts@ustc.edu.cn

JIANFEI WANG

DEPARTMENT OF MATHEMATICS

University of SCIENCE AND TECHNOLOGY OF CHINA

HEFEI, ANHUI, 230026

CHINA

wangjf@mail.ustc.edu.cn 